\title{
Classification of BZCAT objects having uncertain types
}

\author{
H.V.Abrahamyan, A.M.Mickaelian†, G.M.Paronyan‡, G.A.Mikayelyan§, and M.V.Gyulzadyanף \\ NAS RA V. Ambartsumian Byurakan Astrophysical Observatory (BAO), Armenia
}

\begin{abstract}
In this work we try to understand some optical properties of blazars having uncertain types (BZU) in BZCAT vs. 5 Catalogue. Cross-correlation with SDSS reveals 43 BZU out of 227 that have spectra in SDSS. We have carried out spectral reclassification for these 43 blazar candidates (BZU) for activity types. As a result, 37 (86\%) objects out of 43 changed their previous type.
\end{abstract}

Keywords: blazar, active galactic nuclei.

\section{Introduction}

Blazars are considered to be the most energetic sources in the Universe. BLL Lac was discovered by the Hoffmeister (Hoffmeister, 1929). The originally discovered source was considered to be a variable star. Later, a thorough study of this source showed that it was extragalactic radio source. Discovered source was a radio source which had optical variability. Nowadays 3,561 blazars are known. The disclosed sources have been published by Massaro et al. (2015) as a general list. In this catalog, Massaro grouped all blazars in four main classes: BZB, BZQ, BZG and BZU. According to the definition, blazars should be radio sources and have optical variability. But information about variability is not complete in this catalogue. Information for optical variability of blazars is given by Abrahamyan et al. (2019).

From BZCAT catalog, we cannot understand which sources are called Blazars. Using this catalogue we plan to take out definition of blazars. But in the first step we must understand properties of different types of Blazars. The most important and very interesting sources are blazars, which are classified as uncertain type (BZU). In this type included sources which have not good spectra, or have united properties which have other types of blazars. So, for better understanding of Blazars we must carry out investigations of all types of Blazars and take out united properties.

For summarize different physical properties of blazars we must understand which properties show different types of blazars (BZU, BZB, BZG and BZU).

This work is dedicated to classification of 43 blazars having uncertain type (BZU) in BZCAT catalogue v.5 (Massaro et al., 2015).

\section{Observational data}

For our investigation we take BZCAT v.5 (Massaro et al., 2015), which includes 3561 blazars. In BZCAT blazars have 4 types (table 1). In table 1 we can see 227 objects out of 3561 have uncertain

\footnotetext{
*abrahamyanhayk@gmail.com, Corresponding author

†'aregmick@yahoo.com

${ }^{\ddagger}$ gurgen@bao.sci.am

$\S$ gormick@mail.ru

『mgyulz@gmail.com
} 
Table 1. Distribution of types of objects in BZCAT.

\begin{tabular}{|c|c|c|}
\hline $\mathbf{N}$ & Type & Numbers \\
\hline 1 & BZB & 1151 \\
\hline 2 & BZG & 274 \\
\hline 3 & BZQ & 1909 \\
\hline 4 & BZU & 227 \\
\hline \hline \multicolumn{2}{|c|}{ All } & $\mathbf{3 5 6 1}$ \\
\hline
\end{tabular}

types of blazars. For our investigation we take these 227 BZU objects.

In the First step we cross-corelated these objects with SDSS (Fan et al., 1999). In results we have 81 identification from which 43 have spectra. Our work is dedicated to these 43 objects. For a better understanding of the properties of BZU objects we cross-correlated with VCV-13 (Véron-Cetty \& Véron, 2010), SDSS and NED (table 2).

Table 2. The list of BZU objects with their activity types and radio morphology.

\begin{tabular}{|c|c|c|c|c|c|}
\hline BZCAT name & SDSS & VCV & \multicolumn{3}{|c|}{ NED } \\
\hline & & Sp. & Object & Radio Morphology & Activity Type \\
\hline & & & Type & Homogenized Classification & Homogenized Classification \\
\hline 5BZUJ0217-0820 & Star & $\mathrm{BL}$ & QSO & & BL Lac \\
\hline 5BZUJ0304+0002 & Star & & QSO & & QSO \\
\hline 5BZUJ0742+3744 & Star & & QSO & & QSO \\
\hline 5BZUJ0840+1312 & Star & $\mathrm{S} 1.2$ & QSO & FR II & Sy 1.2 \\
\hline 5BZUJ0849+5108 & Star & S1n & QSO & & Flat-Spectrum Radio Source \\
\hline 5BZUJ0856+0140 & Star & $\mathrm{BL}$ & $\mathrm{G}$ & & BL Lac \\
\hline 5BZUJ0909+4253 & Star & S2 & QSO & Core-Dominated & CSS \\
\hline 5BZUJ0933+0003 & Galaxy & BL? & RadioS & & \\
\hline 5BZUJ0954+5719 & Star & & QSO & & QSO \\
\hline 5BZUJ1000+2233 & Star & S2 & QSO & & \\
\hline 5BZUJ1021+4523 & Star & & $\mathrm{G}$ & radio jet & Flat-Spectrum Radio Source \\
\hline 5BZUJ1030+3102 & Galaxy & S1.5 & G & radio jet & Flat-Spectrum Radio Source \\
\hline 5BZUJ1033+0711 & Galaxy & & RadioS & & Flat-Spectrum Radio Source \\
\hline 5BZUJ1051+4644 & Star & & QSO & & Flat-Spectrum Radio Source \\
\hline 5BZUJ1058+0133 & Star & $\mathrm{HP}$ & QSO & radio jet & BL Lac \\
\hline 5BZUJ1059+4051 & Star & & RadioS & & \\
\hline 5BZUJ1153+5831 & Galaxy & S1.5 & QSO & & Sy 1.5 \\
\hline 5BZUJ1208+6121 & Galaxy & BL? & G & & BL Lac \\
\hline 5BZUJ1225+4834 & Star & & QSO & & QSO \\
\hline 5BZUJ1238+5325 & Star & S1 & $\mathrm{G}$ & & Sy 1 \\
\hline 5BZUJ1257+0024 & Star & & QSO & & QSO \\
\hline 5BZUJ1302+5748 & Star & & QSO & & Flat-Spectrum Radio Source \\
\hline 5BZUJ1310+3220 & Star & HP & QSO & & Flat-Spectrum Radio Source \\
\hline 5BZUJ1345+4125 & Star & & QSO & & \\
\hline 5BZUJ1345+5332 & Galaxy & S1 & $\mathrm{G}$ & & \\
\hline 5BZUJ1347+3012 & Star & & QSO & & QSO \\
\hline 5BZUJ1353+0443 & Star & & QSO & & Flat-Spectrum Radio Source \\
\hline 5BZUJ1431-0052 & Star & & QSO & & QSO \\
\hline 5BZUJ1435+2021 & Star & BL? & $\mathrm{G}$ & & Flat-Spectrum Radio Source \\
\hline 5BZUJ1448+0402 & Star & $\mathrm{S} 2$ & $\mathrm{G}$ & & Flat-Spectrum Radio Source \\
\hline 5BZUJ1449+4221 & Galaxy & $\mathrm{S} 2$ & QSO & & Flat-Spectrum Radio Source \\
\hline 5BZUJ1458+0416 & Star & & QSO & & Flat-Spectrum Radio Source \\
\hline 5BZUJ1511+0518 & Galaxy & S1 & $\mathrm{G}$ & & Flat-Spectrum Radio Source \\
\hline 5BZUJ1536+3742 & Galaxy & & G & & \\
\hline 5BZUJ1550+1120 & Star & S1.5 & QSO & & Flat-Spectrum Radio Source \\
\hline 5BZUJ1557+3304 & Star & & QSO & radio jet & $\mathrm{QSO}$ \\
\hline 5BZUJ1602+2646 & Galaxy & S2 & $\mathrm{G}$ & & Flat-Spectrum Radio Source \\
\hline 5BZUJ1603+1554 & Galaxy & & $\mathrm{G}$ & & Flat-Spectrum Radio Source \\
\hline 5BZUJ1618+2159 & Star & S1 & QSO & & \\
\hline 5BZUJ1633+2112 & Galaxy & S1 & QSO & & \\
\hline 5BZUJ1706+3214 & Star & & RadioS & & \\
\hline 5BZUJ2156-0037 & Star & $\mathrm{BL}$ & QSO & & Flat-Spectrum Radio Source \\
\hline 5BZUJ2327+1524 & Galaxy & S1 & $\mathrm{G}$ & & Flat-Spectrum Radio Source \\
\hline
\end{tabular}


Using table 2 we can conclude the following:

- In SDSS: 13 objects are "galaxies" (extended objects) and 30 are "stars" (point-like objects),

- In VCV-13: 6 objects are BL or BL?, 2 objects are HP (HPQ), 6 objects are Sy1, 1 object is Sy1.2, 3 objects are Sy1.5, 1 object is Sy1n (Narrow Line Seyfert 1), 5 objects are Sy2, and for 19 objects we do not have any information,

- In NED: 13 objects are galaxies, 26 objects are quasars and 4 objects are RadioS (radio sources). Among these objects we have 4 BL Lac, 18 FSS (Flat-Spectrum Radio Source), 1 CSS (Compact Steep Spectrum), 1 Sy1, 1 Sy1.2, and 1 Sy 1.5,

- In NED we have radio morphology: 4 objects have radio jets, 1 object is FRII and 1 object is core-dominated radio object.

In Figure 1, we give redshift distribution of BZU and distribution of 43 objects, which have spectra in SDSS.

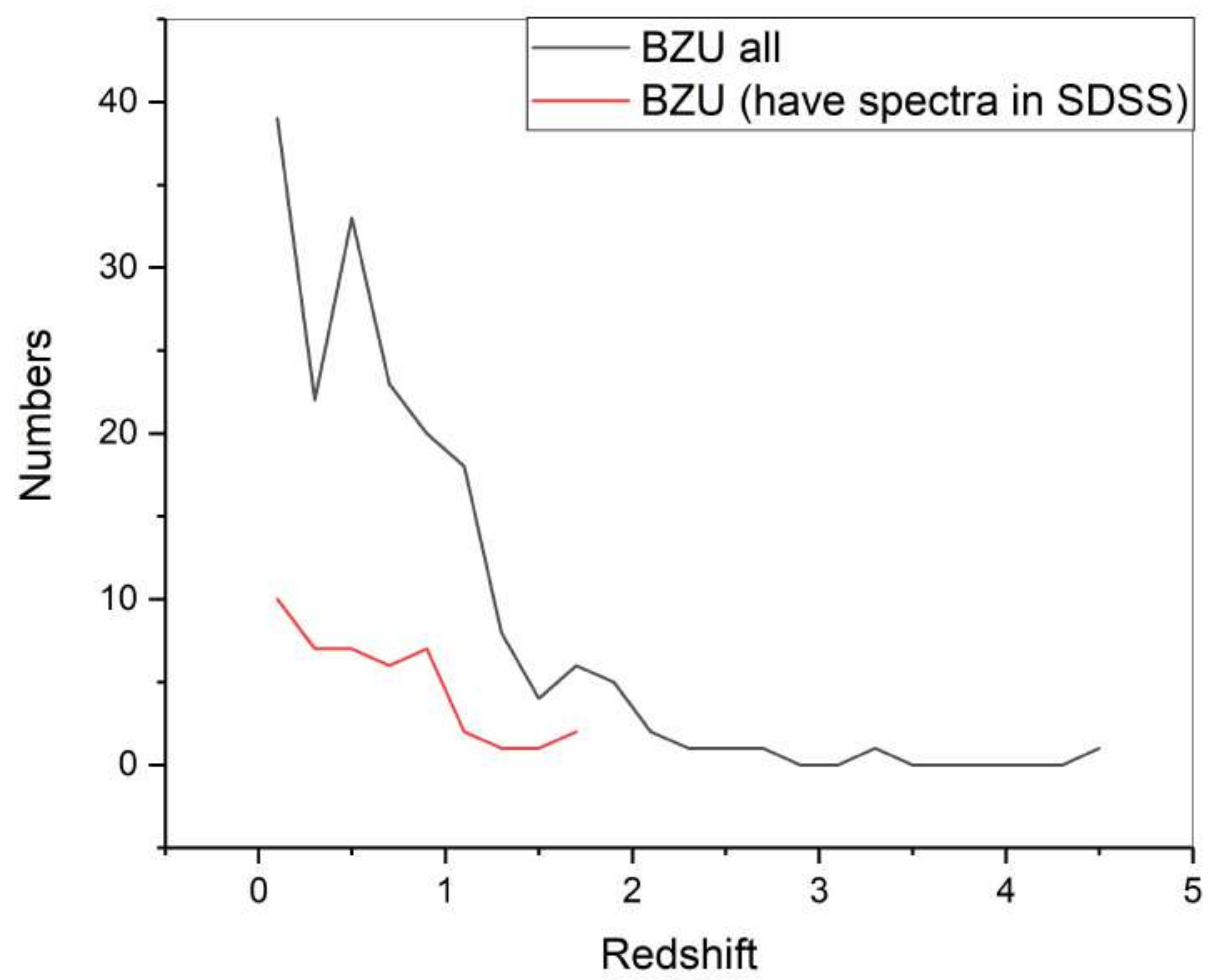

Figure 1. Redshift distribution of BZU objects.

In figure $1 \mathrm{BZU}$ source mainly have 0 until 2.2 redshift and our studied sources have 0 until 1.75 redshift. For these 43 sources we have done classification using SDSS spectra.

For understanding some physical properties of blazars we use the paper Abrahamyan et al. (2019). In this paper calculated absolute magnitude for all blazars. In Figure 2, we give graphs of the absolute magnitude versus redshift.

In Figure 2 our investigated sources have -21 to -25 absolute magnitudes.

So, using 43 SDSS spectra for our sources, we have carried out optical classification. 


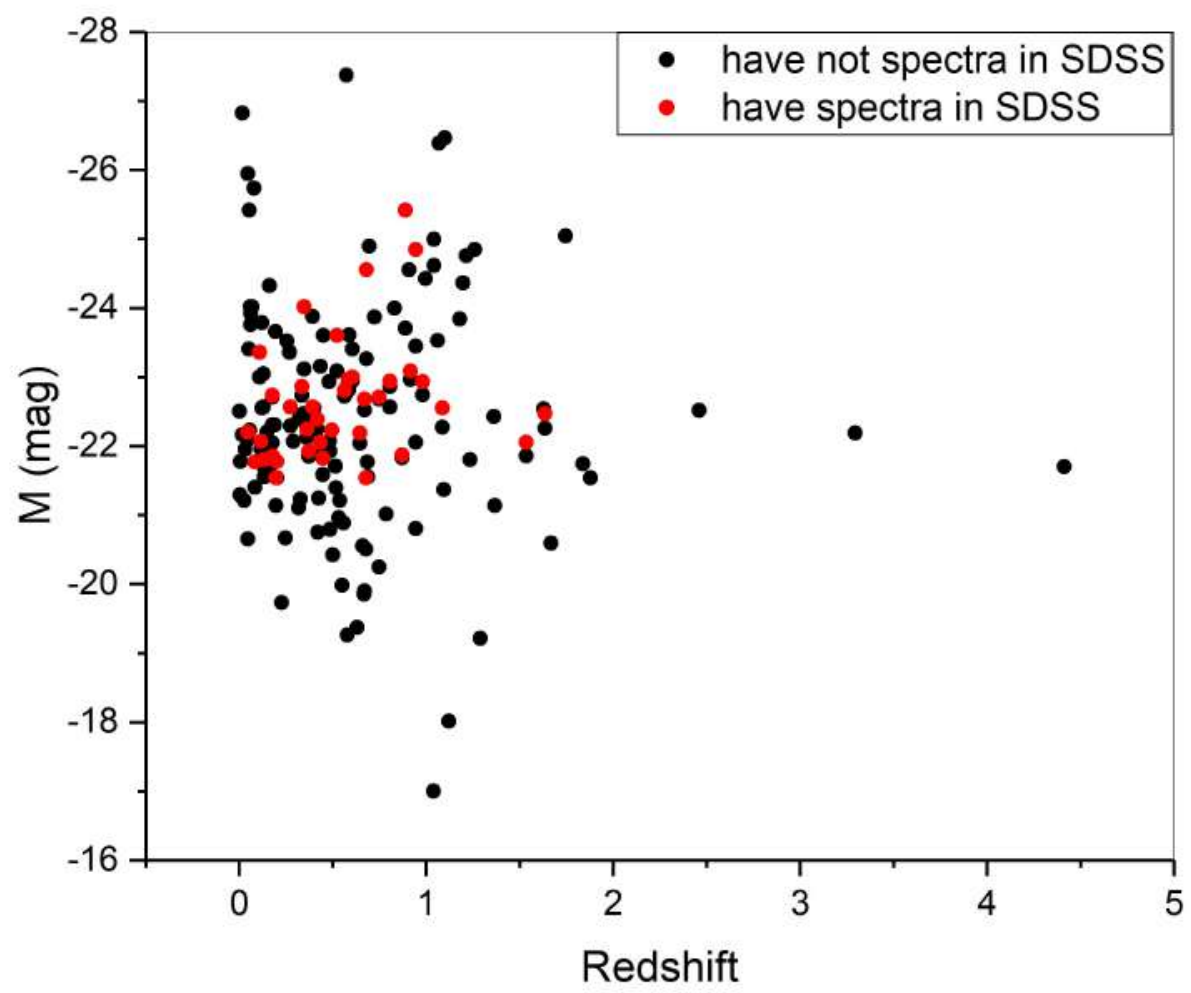

Figure 2. Absolute magnitude vs. redshift.

\section{Classification method and results}

We have used several methods for classification of our spectra (Mickaelian et al., 2018);

- By eye (taking into account all features and effects)

- By diagnostic diagram using $[\mathrm{OIII}] / \mathrm{H}_{\beta}$ and $[\mathrm{NII}] / \mathrm{H}_{\alpha}$ ratios (Reines et al., 2013),

- By diagnostic diagram using $[\mathrm{OIII}] / \mathrm{H}_{\beta}$ and $[\mathrm{SII}] / \mathrm{H}_{\alpha}$ ratios (Reines et al., 2013),

- By diagnostic diagram using $[\mathrm{OIII}] / \mathrm{H}_{\beta}$ and $[\mathrm{OI}] / \mathrm{H}_{\alpha}$ ratios (Reines et al., 2013),

We have done classification only by eye, because we have not enough information for diagnostic diagrams from the spectra.

We started the studying of spectra with identifications of spectral lines. We have used only lines having intensities 3 sigma over the noise level.

In Figure 3 we give 4 spectra out of 43 our investigated blazars.

In order to do classification, we need to consider the classification Massaro et al. (2015):

- BZB: BL Lac objects, used for AGNs with a featureless optical spectrum, or having only absorption lines of galactic origin and weak and narrow emission lines (Massaro et al., 2015);

- BZG objects, usually reported as BL Lac objects in the literature, but having a spectral energy distribution (SED) with a significant dominance of the galactic emission over the nuclear one (Massaro et al., 2015); 

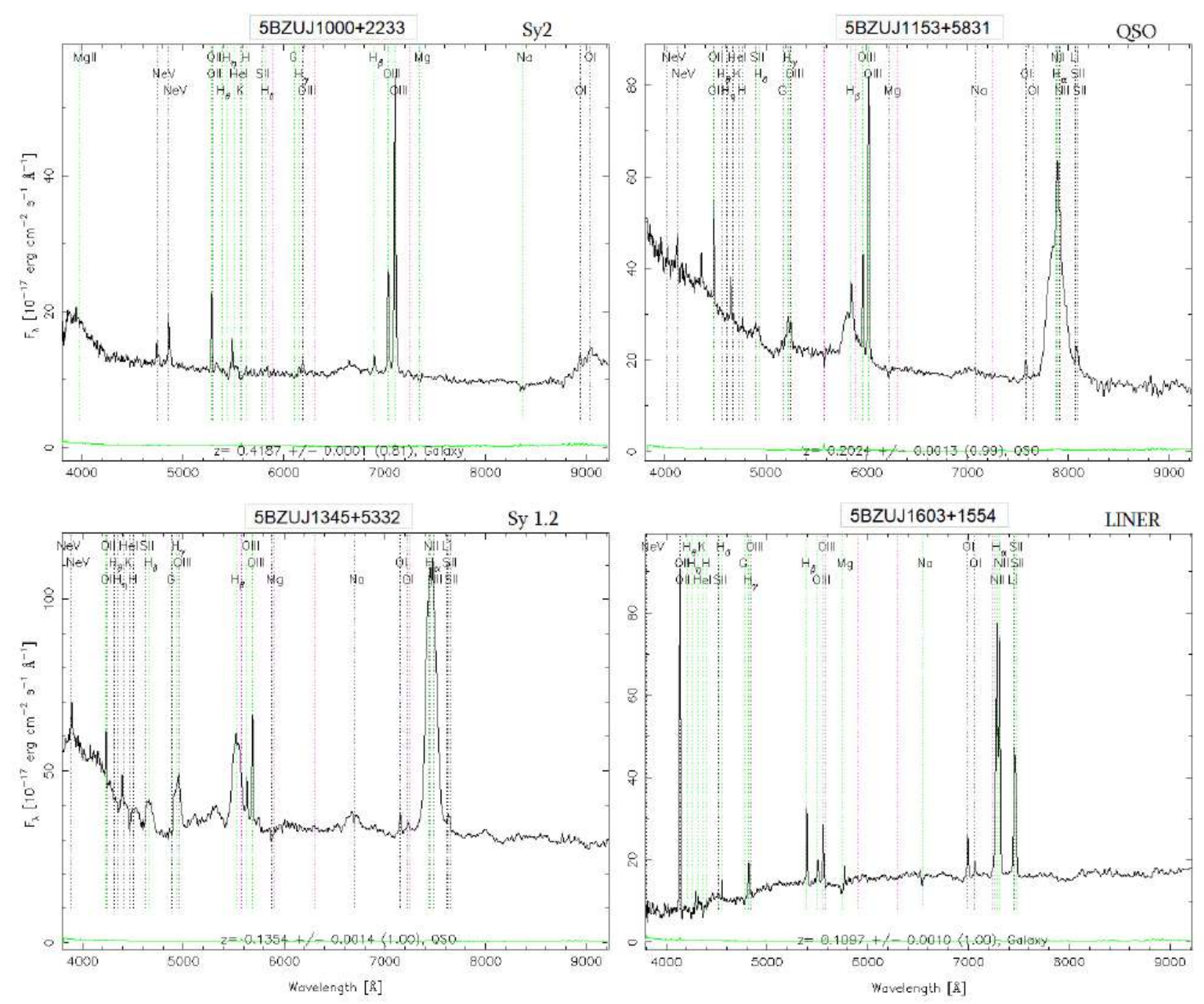

Figure 3. Examples of spectra.

- BZQ: Flat Spectrum Radio Quasars, with an optical spectrum showing broad emission lines and dominant blazar characteristics (Massaro et al., 2015).

Using classification of BZCAT given in Massaro et al. (2015). we have carried out classification of 43 sources which have uncertain type.

So, 37 BZU objects from 43 changed their classification to BZQ, BZG and BZG. In table 3 we give the new classification and redshifts from SDSS.

In table 3 we give old and new classification, and give activity type using SDSS spectra. And we give redshift form catalogue BZCAT v. 5, NED and SDSS. For 5 sources we checked and corrected redshift and for 4 sources is given by SDSS and for 1 source is given by BZCAT.

\section{Conclusion}

So, having optical spectra of 43 BZU, we reclassified these objects. As the main results we have:

1) $37(86 \%)$ objects from 43 changed classification (table 4$)$.

2) In table 3 we give information of redshift from BZCAT, SDSS and NED. For 5 objects that numbers are different (5BZUJ0933+0003, 5BZUJ1051+4644, 5BZUJ1058+0133, 5BZUJ1302+5748, 5BZUJ2156-0037). We checked and corrected redshift and for 4 (5BZUJ0933+0003, 5BZUJ1051+4644, 5BZUJ1302+5748, 5BZUJ2156-0037) sources is given by SDSS and for 1 (5BZUJ1058+0133) sources is given by BZCAT. 
Table 3. New classification of BZU objects.

\begin{tabular}{|c|c|c|c|c|c|c|}
\hline \multicolumn{2}{|c|}{ BZCAT v.5 } & \multirow{2}{*}{\multicolumn{2}{|c|}{ Our classifaction }} & \multicolumn{3}{|c|}{ Redshift } \\
\hline Source name & Type & & & BZCAT & NED & SDSS \\
\hline 5BZUJ0217-0820 & BZU & NLQSO & $\overline{B Z Q}$ & 0.607 & 0.606538 & 0.60654 \\
\hline 5BZUJ0304+0002 & BZU & QSO1.2 & BZQ & 0.564 & 0.56417 & 0.56366 \\
\hline 5BZUJ0742+3744 & $\overline{B Z U}$ & QSO1.5 & BZQ & 0.806 & 0.806274 & 0.80574 \\
\hline 5BZUJ0840+1312 & $\mathrm{BZU}$ & QSO1.2 & BZQ & 0.681 & 0.6808 & 0.68037 \\
\hline 5BZUJ0849+5108 & $\mathrm{BZU}$ & QSO & BZQ & 0.583 & 0.584701 & 0.58345 \\
\hline 5BZUJ0856+0140 & $\overline{B Z U}$ & Unknown & BZU & 0.448 & 0.448184 & 0.44807 \\
\hline 5BZUJ0909+4253 & $\mathrm{BZU}$ & QSO & BZQ & 0.670 & 0.669915 & 0.67041 \\
\hline 5BZUJ0933+0003 & $\mathrm{BZU}$ & Unknown & BZU & $0^{b}$ & & $0.71107^{a}$ \\
\hline 5BZUJ0954+5719 & BZU & QSO & $\mathrm{BZQ}$ & 0.981 & 0.981193 & 0.98121 \\
\hline 5BZUJ1000+2233 & BZU & Sy2.0 & BZG & 0.419 & 0.418732 & 0.41874 \\
\hline 5BZUJ1021+4523 & $\overline{B Z U}$ & QSO & $\overline{B Z Q}$ & 0.364 & 0.36388 & 0.36437 \\
\hline 5BZUJ1030+3102 & $\mathrm{BZU}$ & QSO & BZQ & 0.178 & 0.1782 & 0.17815 \\
\hline 5BZUJ1033+0711 & $\overline{B Z U}$ & Unknown & BZU & 1.535 & & 1.52948 \\
\hline 5BZUJ1051+4644 & BZU & Unknown & BZU & $0^{b}$ & $1.419418^{b}$ & $0.00005^{a}$ \\
\hline 5BZUJ1058+0133 & BZU & BLL & BLB & $0.890^{a}$ & $0.89^{a}$ & $0.3823^{b}$ \\
\hline 5BZUJ1059+4051 & BZU & QSO & $\overline{B Z Q}$ & 1.746 & & 1.75049 \\
\hline 5BZUJ1153+5831 & BZU & QSO & BZQ & 0.202 & 0.202439 & 0.2024 \\
\hline 5BZUJ1208+6121 & $\overline{B Z U}$ & Abs & $\overline{B Z G}$ & 0.275 & 0.274783 & 0.27479 \\
\hline 5BZUJ1225+4834 & $\mathrm{BZU}$ & QSO & BZQ & 0.647 & 0.646553 & 0.64687 \\
\hline 5BZUJ1238+5325 & $\overline{B Z U}$ & QSO1.2 & $\overline{B Z Q}$ & 0.348 & 0.347506 & 0.34684 \\
\hline 5BZUJ1257+0024 & $\mathrm{BZU}$ & QSO & BZQ & 1.259 & 1.260971 & 1.25808 \\
\hline 5BZUJ1302+5748 & BZU & Unknown & BZU & $1.088^{b}$ & $1.088^{b}$ & $0.83066^{a}$ \\
\hline 5BZUJ1310+3220 & $\overline{B Z U}$ & QSO & $\overline{B Z Q}$ & 0.997 & 0.998007 & 0.99725 \\
\hline 5BZUJ1345+4125 & $\mathrm{BZU}$ & QSO & BZQ & 0.916 & 0.916932 & 0.91654 \\
\hline 5BZUJ1345+5332 & $\overline{B Z U}$ & Sy1.2 & BZG & 0.135 & 0.135406 & 0.13537 \\
\hline 5BZUJ1347+3012 & $\mathrm{BZU}$ & Sy1.5 & BZG & 0.118 & 0.11785 & 0.11784 \\
\hline 5BZUJ1353+0443 & BZU & QSO & BZQ & 0.523 & 0.522821 & 0.5234 \\
\hline 5BZUJ1431-0052 & $\overline{B Z U}$ & QSO & BZQ & 1.635 & 1.633083 & 1.63687 \\
\hline 5BZUJ1435+2021 & $\mathrm{BZU}$ & Em & BZG & 0.748 & 0.748 & 0.74768 \\
\hline 5BZUJ1448+0402 & $\mathrm{BZU}$ & Em & $\overline{B Z G}$ & 0.871 & 0.8712 & 0.8712 \\
\hline 5BZUJ1449+4221 & BZU & Sy2.0 & BZG & 0.179 & 0.1783 & 0.17867 \\
\hline 5BZUJ1458+0416 & BZU & Em & BZG & 0.392 & 0.391547 & 0.39154 \\
\hline 5BZUJ1511+0518 & $\overline{B Z U}$ & Sy2.0 & BZG & 0.084 & 0.084 & 0.08452 \\
\hline 5BZUJ1536+3742 & $\mathrm{BZU}$ & Em & BZG & 0.679 & 0.679211 & 0.67911 \\
\hline 5BZUJ1550+1120 & $\overline{B Z U}$ & Sy1.5 & $\overline{B Z G}$ & 0.436 & 0.43598 & 0.43567 \\
\hline 5BZUJ1557+3304 & $\mathrm{BZU}$ & QSO & BZQ & 0.943 & 0.944472 & 0.94962 \\
\hline 5BZUJ1602+2646 & BZU & LINER & BZG & 0.372 & 0.371657 & 0.37171 \\
\hline 5BZUJ1603+1554 & $\mathrm{BZU}$ & LINER & BZG & 0.110 & 0.109866 & 0.10971 \\
\hline 5BZUJ1618+2159 & $\mathrm{BZU}$ & QSO & BZQ & 0.336 & 0.334828 & 0.3348 \\
\hline 5BZUJ1633+2112 & $\overline{B Z U}$ & Sy1.8 & BZG & 0.198 & 0.198156 & 0.1982 \\
\hline 5BZUJ1706+3214 & $\mathrm{BZU}$ & QSO & BZQ & 1.070 & & 1.06979 \\
\hline 5BZUJ2156-0037 & $\mathrm{BZU}$ & Unknown & $\mathrm{BZU}$ & $0.495 ?^{b}$ & $0.495^{b}$ & $2.23931^{a}$ \\
\hline 5BZUJ2327+1524 & $\mathrm{BZU}$ & QSO & BZQ & 0.046 & 0.045717 & 0.04581 \\
\hline
\end{tabular}

(a) Right measurement.

(b) Wrong measurement.

3) Using SDSS spectra we have carried out classification in optical range. In table 5 we give 
Table 4. New classification of BZU.

\begin{tabular}{|c|c|c|c|}
\hline N & Old & New & Numbers \\
\hline 1 & BZU & BZB & $1(2 \%)$ \\
\hline 2 & BZU & BZG & $14(33 \%)$ \\
\hline 3 & BZU & BZQ & $22(51 \%)$ \\
\hline 4 & BZU & BZU & $6(14 \%)$ \\
\hline \hline \multicolumn{3}{|c|}{ All } & $\mathbf{4 3 ( 1 0 0 \% )}$ \\
\hline
\end{tabular}

information for this classification.

Table 5. Spectral classification using SDSS spectra

\begin{tabular}{|c|c|}
\hline Activity Type & Numbers \\
\hline Abs & 1 \\
\hline BLL & 1 \\
\hline Em & 4 \\
\hline LINER & 2 \\
\hline NLQSO & 1 \\
\hline QSO & 17 \\
\hline QSO 1.2 & 3 \\
\hline QSO 1.5 & 1 \\
\hline Sy 1.2 & 1 \\
\hline Sy 1.5 & 2 \\
\hline Sy 1.8 & 1 \\
\hline Sy 2.0 & 3 \\
\hline Unknown & 6 \\
\hline \hline Total & 43 \\
\hline
\end{tabular}

In VCV-13 catalogue, if the absolute magnitude is more than -22.25 then the sources are classified as quasars in Véron-Cetty \& Véron (2010). So, using that, among our sources we had classification QSO 1.2 and QSO 1.5. If these sources have absolute magnitude less than -22.25 , we classify them as Sy1.2 and Sy1.5.

QSO 1.2 and QSO 1.5 have the same properties which have Sy 1.2 and Sy1.5, and according to VCV catalogue there is only absolute magnitude limit -22.25 .

\section{References}

Abrahamyan H. V., Mickaelian A. M., Mikayelyan G. A., Paronyan G. M., 2018, ComBAO, 2 (LXV), 1

Abrahamyan H. V., Mickaelian A. M., Paronyan G. M., Mikayelyan G. A., 2019, AN, 5, 437

Fan X., Strauss M. A., Schneider D. P., et al. 1999, AJ, 1, 118

Hoffmeister C., 1929, AN, 233, 236

Massaro E., Alessandro M., Cristina L., 2015, Ap.SS, 4, 357

Mickaelian A. M., Harutyunyan G. S., Sarkissian A., 2018, Astronomy Letters, 6, 44

Paronyan G. M., Mickaelian A. M., Harutyunyan G. S., Abrahamyan H. V., Mikayelyan G. A., 2019, Astrophysics, 62, 147

Reines A. E., Greene J. E., Geha M., 2013, AJ, 2, 755

Véron-Cetty M.-P., Véron P., 2010, A\&A, A10, 518 